\title{
Predictors of Extubation Failure In Thymomatous Myasthenia Gravis Patient After Thymectomy: Ten Years of Data From A Tertiary Teaching Hospital In Mainland China
}

\author{
Anqi Du \\ Peking university people's hospital https://orcid.org/0000-0002-7146-9637

\section{Xiao Li} \\ Peking University People's Hospital \\ Youzhong An ( $\nabla$ bjrmyy_icu@163.com ) \\ Peking university people's hospital
}

\section{Research}

Keywords: myasthenia gravis, thymectomy, post-thymectomy myasthenic crisis, extubation, risk factors

Posted Date: March 2nd, 2021

DOI: https://doi.org/10.21203/rs.3.rs-277082/v1

License: (c) (i) This work is licensed under a Creative Commons Attribution 4.0 International License.

Read Full License 


\section{Abstract}

Background: Thymectomy was consider to be the first line therapy for Patients with thymoma-associated myasthenia gravis(T-MG). But weaning is still a big challenge for MG patents, especially after surgery. The ideal time for extubation and the risk factors of extubation failure is uncertain. This study was to investigate the risk factors of extubation failure in T-MG patients after thymectomy.

Methods: Retrospective review of consecutive T-MG patients admitted in intensive care unit after thymectomy and need mechanical ventilation at least 6h between January 2010 and December 2019. Weaning was according to a unified process. Extubation failure was defined as need for reintubation within $48 \mathrm{~h}$ after extubation or death during ventilation. Univariate logistic regression was used to identify predictors of extubation failure.

Results: 38 patients were included. 13 patients (34.2\%) were extubation failure and with longer ventilation duration (153 vs. 29 h, $p<0.05$ ) and hospital stay (26 vs. 13 days, $p<0.05)$. Univariate regression shows the ratio of transsternal thymectomy, the incident of never or diaphragm injury during the surgery or the PTMC, atelectasis, pneumonia during ventilation $(p<0.05)$. Furthermore, even passed $S B T$, a higher HR, RR and lower SpO2 also indicate high risk of exutbation failure $(\mathrm{p}<0.05)$.

Conclusions: Extubation failure is relatively common in T-MG patients after thymectomy. Minimizing surgical trauma may contributes to successful extubation. But the incidence of PTMC or pulmonary compliances during ventilation was associate with high risk of extubation failure. A higher HR, RR and lower SpO2 after SBT and also can be a reliable indicator.

\section{Introduction}

Myasthenia gravis(MG) is an autoimmune disorder characterized by defective neuromuscular junction transmission due to antibodies directed against the postsynaptic acetylcholine receptor (AChR) $[1,2]$. Thirty percent of MG patients can develop fluctuating degree of neuromuscular respiratory weakness. Thymomas can occur in 10\%-30\% MG patients[3], which was considered to be a negative prognostic factor for MG as more serious symptoms and lower reactivity to first line treatments[4-6]. Although it has been clear that thymectomy can improve clinical symptoms all patients with thymoma-associated MG (T$M G)$, it may take weeks or months to achieve remission. However, nearly $11.5-18.2 \%$ patients can be worse by suffering post-thymectomy myasthenic crisis (PTMC) [7], which was defined by the occurrence of neuromuscular respiratory failure requiring mechanical ventilation (MV). It can develop from the weakness of either respiratory muscles, upper airway muscles, or both. Thus, some patients still need ventilation and will remain for more than a week after surgery $[8,9]$, which increase the risk of pneumonia, atelectasis, ventilator-induced diaphragmatic dysfunction[10,11], and prolong ventilation[12]. In order to avoid these vicious circle, MG patients should be weaned as soon as possible. General, weaning accounts for over $40 \%$ of the total MV time and can be successful in most cases $[13,14]$ But it is much 
more complicated and challenging in MG patients, $25-40 \%$ will fail weaning $[15,16]$. In addition, there is no set approach and little is known about the risk factors of extubation failure for these patients.

The main purposes of this retrospective study are to evaluate the risk of extubation failure in T-MG patients after thymectomy and determine which clinical factors may predict the outcome extubation.

\section{Materials And Method}

\section{Study design and patients}

This study was approved by the Institutional Ethics Review Board of Peking University People's Hospital. Informed consent was waived due to the retrospective nature of the study.

Adult T-MG patients who underwent surgical therapy in the intensive care unit(ICU) of Peking University People's Hospital between January 2010 and December 2019 were retrospectively collected.

Patients who met the following criteria were excluded :1) extubate immediately after surgery, 2) cumulative duration of postoperative mechanical ventilation less than $6 \mathrm{~h}$. The diagnosis of MG was confirmed through clinical presentation, positive AChR antibodies and/or single-fiber electromyography results[7]. MG clinical severity was graded by Osserman classification. A total 38 patients were included.

All patients maintained with endotracheal intubation and standard ventilation in ICU after thymectomy by unilateral video-assisted thoracoscopic (VATS) or the open transsternal (OT). A multidisciplinary protocol was required and continuous dynamic monitoring of arterial blood pressure, heart rate and finger oxygen saturation was performed in ICU. X-ray films were reviewed for the presence of atelectasis or pneumonia. Blood gas analysis was performed every $12 \mathrm{~h}$. According to the patient's condition, the attending and respiratory therapist responsible for the patients reduced the amount of respiratory support gradually. Tracheostomy should be considered when the duration of intubation was $\geq 2$ weeks. The decision for extubation was based on the result of spontaneous breath trail(SBT) and the subjective assessment. If failure, an advance ventilation support should be given immediately and reevaluated the next day. Or the patients can be extubated as long as the airway was patency and cough was powerful. The details were show as blow. Noninvasive ventilation(NIV) can be used after extubation to prevent reintubation or to complete weaning in patients with persistent requirements of ventilator support.

\section{SBT implementation method}

Low-level pressure support ventilation was used in SBT, which pressure support is blow $8 \mathrm{~cm} \mathrm{H}_{2} \mathrm{O}$, positive end expiratory pressure(PEEP) was 4 to $6 \mathrm{~cm} \mathrm{H}_{2} \mathrm{O}$, and the fraction of inspired oxygen $\left(\mathrm{FiO}_{2}\right)$ was below $40 \%$, and lasting for $30 \mathrm{~min}$ to $120 \mathrm{~min}$.

\section{Sbt Failure Criteria}


Patients who showed changes in mental status, including anxiety, restlessness, delirium, and somnolence, and/or who met the following criteria [13][8]:

Arterial oxygen pressure $\left(\mathrm{PaO}_{2}\right) \leq 50-60 \mathrm{mmHg}$, arterial carbon dioxide pressure $\left(\mathrm{PaCO}_{2}\right) \geq 50 \mathrm{mmHg}$ or $\Delta \geq 8 \mathrm{mmHg}$; respiratory rate (RR) $\geq 35 / \mathrm{min}$ or $\Delta \geq 50 \%$, heart rate $(\mathrm{HR}) \geq 140$ beats $/ \mathrm{min}$ or $\Delta \geq 20 \%$, systolic blood pressure $\geq 180 \mathrm{~mm} \mathrm{Hg}$ or $\geq \Delta 20 \%$, systolic blood pressure $<90 \mathrm{~mm} \mathrm{Hg}$, or arrhythmia.

Exutbation failure[17] was defined as need for reintubation within 48h after extubation or death during ventilation.

According to the outcome of extubation, the patients were divided into successful and failure groups. The duration of MV was the time between the end of surgery to extubation or death during MV. We collected demographic information, coexistent illness, duration of the disease was defined as the period between the onset of initial symptoms to hospital admission, tumor size evaluated by CT scans, surgical approaches, operation time, blood loss during the operation, Masaoka Koga stage[18], and incidence of PTMC and acute physiology and chronic health evaluation II (APACHEII) score at the first day in ICU. Data on vital signs and blood gases, maximal inspiratory pressure(MIP) and diagram ultrasound before extubation also be gathered. Besides, all complications during hospitalization were recorded, epically pulmonary compliances (pneumonia, atelectasis).

\section{Statistical analysis}

Collected data were recorded into a database which were subsequently analyzed with SPSS (Version 25 , SPSS Inc., Armonk, NY, USA). Comparison of two groups of continuous variables was achieved with Mann-Whitney $U$ test. Comparison of categorical variables was performed using $\chi 2$ test or Fisher's exact test as appropriate. Backward univariate logistic models were used to investigate potential risk factors. All tests were two-sided and $\mathrm{P}<0.05$ was considered as statistically significant.

\section{Results}

During the study period, the data of 43 patients who met our inclusion criteria were collected from medical record system.

6 patients received MV less than $6 \mathrm{~h}$, and 1 patients underwent tracheostomy without any preceding extubation attempts were excluded from the analysis. Finally, 38 patients were included in our study, 11 (34.2\%)were need reintubation and 2 were death while ventilation.

Table 1. Baseline characteristics 


\begin{tabular}{|lllll|}
\hline Parameter & Success $(\mathrm{n}=25)$ & Failure $(\mathrm{n}=13)$ & $\mathrm{Z} / \mathrm{X} 2$ & P-value \\
\hline Age(years), M (p25, p75) & $54(45,66.0)$ & $40(35,58)$ & -1.988 & 0.047 \\
\hline Male, $\mathrm{n}(\%)$ & $15(60.0)$ & $8(61.5)$ & 0.008 & 0.927 \\
\hline BMI(kg/m²), M (p25, p75) & $24.4(22.7,27.8)$ & $22.5(20.8,25.0)$ & -1.478 & 0.139 \\
\hline Complications, $\mathrm{n}(\%)$ & & & & \\
\hline Hypertension & $6(24)$ & $2(15.2)$ & 0.039 & 0.843 \\
\hline Pulmonary disease & $0(0.0)$ & $2(15.4)$ & - & 0.111 \\
\hline Smoking, $\mathrm{n}(\%)$ & $7(28.0)$ & $6(42.6)$ & 0.576 & 0.448 \\
\hline Duration of the disease(day), M (p25, p75) & $90(30,365)$ & $30(23,150)$ & -1.273 & 0.203 \\
\hline Osserman Classification (IIb + III + IV), n (\%) & $15(60.0)$ & $10(76.9)$ & 0.466 & 0.495 \\
\hline Use of pyridostigmine (mg), M (p25, p75) & $12(48.0)$ & $10(45.4)$ & 2.935 & 0.087 \\
\hline APACHII, M (p25, p75) & $16(16,18)$ & $19(15,23)$ & -1.637 & 0.102 \\
\hline BMI, Body Mass Index; VC, Vital Capacity; APACHII, Acute Physiology and Chronic Health Evaluation. \\
\hline
\end{tabular}

\section{Baseline Clinical And Demographic Characteristics}

The median age of patients in our study was 52 years (range,40-66 years), and the female to male ratio was $1: 1.5$. Nearly $65.8 \%$ of them were in late stage (Osserman Classification IIb -IV), the median disease duration was 90 days (range, $20-225$ days). $57.9 \%$ of them were treated with pyridostigmin, $3(7.9 \%)$ patients were also receiving steroids and $3(7.9 \%)$ were receiving intravenous immunoglobulin. None of them had previous history of myasthenic crisis. 2 patients had a tracheostomy. The baseline characteristics of two group were depicted in Table 1. Most of the baseline variables did not differ much between this two group, except for the age, the weaning failure group was older $(P<0.05)$.

\section{Characteristics Of Perioperative And Pathological Data}

The mean diameter of the tumor was $5 \mathrm{~cm}$ (range $3-8 \mathrm{~cm}$ ).

28(73.7\%) patients with locally advanced tumors (Masaoka stage II-IV).

VAST thymectomy was performed in 30 patients, but 3 of them were converted to OT. And OT was more in weaning failure group $(53.8 \%$ vs. $16 \%, p<0.05), 63.6 \%(7 / 11)$ of them were weaning failure. The details are shown in Table 2. The ratio of phrenic never or diaphragm injury was higher in the extubation failure group $(61.5 \%$ vs. $28.0 \%, p<0.05)$. The incident of postoperative complications including PTMC, 
pulmonary compliance was in $8(21.1 \%)$ and $16(42.1 \%)$, and was much higher in weaning failure group $(p<0.05)$.

Table 2

Perioperative, and pathological data

\begin{tabular}{|lllll|}
\hline Parameter & Success $(\mathrm{n}=25)$ & Failure $(\mathrm{n}=13)$ & $Z / \chi 2$ & $P$-value \\
\hline Size of thymomas $(\mathrm{cm}), \mathrm{M}(\mathrm{p} 25, \mathrm{p} 75)$ & $5(3,6)$ & $7(3,9)$ & -0.697 & 0.486 \\
\hline Operative blood loss $(\mathrm{ml}), M(p 25, p 75)$ & $50(20,100)$ & $125(24,200)$ & -1.770 & 0.077 \\
\hline Operation time (min), $M(p 25, p 75)$ & $180(135,255)$ & $211(185,345)$ & -1.758 & 0.079 \\
\hline Surgical procedure (OT), $\mathrm{n}(\%)$ & $4(16.0)$ & $7(53.8)$ & 4.258 & 0.039 \\
\hline Phrenic never or diaphragm injury, $\mathrm{n}(\%)$ & $7(28.0)$ & $8(61.5)$ & 4.027 & 0.045 \\
\hline Masaoka stage (II-V), n (\%) & $18(72.0)$ & $10(76.9)$ & 0.107 & 0.744 \\
\hline PTMC, $\mathrm{n}(\%)$ & $2(8.0)$ & $6(46.2)$ & 5.371 & 0.020 \\
\hline Atelectasis and/or pneumonia., $\mathrm{n}(\%)$ & $6(24.0)$ & $10(76.9)$ & 9.827 & 0.002 \\
\hline OT, open transsternal; PTMC, Post-Operative Myasthenic Crisis. & & \\
\hline
\end{tabular}

\section{Characteristics Of Clinical Data Before Extubation}

PSV was the weaning method in 36 patients (94.7\%), T piece was only used in 2 patients with tracheotomy. As described in Table 3, patients in the extubation failure group had higher HR, RR and lower $\mathrm{SpO}_{2}$ than patients in the successful weaning group after SBT. These differences were statistically significant $(p<0.05)$. 
Table 3

Variables before weaning.

\begin{tabular}{|c|c|c|c|c|}
\hline Parameter, $M(p 25, p 75)$ & Success $(n=25)$ & Failure(n = 13) & $Z / \chi^{2}$ & $P$-value \\
\hline MBP(mmHg) & $86(70,102)$ & $74(65,93)$ & -1.868 & 0.062 \\
\hline $\mathrm{HR}(\mathrm{bpm})$ & $89(80,96)$ & $104(96,128)$ & -2.773 & 0.006 \\
\hline RR(bpm) & $20(18,21)$ & $23(19,28)$ & -2.158 & 0.020 \\
\hline $\mathrm{SpO}_{2}$ & $100(98,100)$ & $96(92,100)$ & -2.273 & 0.023 \\
\hline $\mathrm{PH}$ & $7.429(7.403,7.450)$ & 7.469(7.422,7.495) & -1.369 & 0.171 \\
\hline $\mathrm{PCO}_{2}\left(\mathrm{cmH}_{2} \mathrm{O}\right)$ & $31.5(28.4,36.9)$ & $34.7(27.9,40.9)$ & -1.209 & 0.227 \\
\hline$P / F$ & $353(265,420)$ & $321(210,371)$ & -1.114 & 0.265 \\
\hline $\mathrm{Hb}$ & $12.1(11.1,13.3)$ & $11.1(10.4,13.1)$ & -0.987 & 0.337 \\
\hline $\operatorname{MIP}\left(\mathrm{cmH}_{2} \mathrm{O}\right)$ & $38(36,41)$ & $33(32,35)$ & -1.875 & 0.061 \\
\hline \multicolumn{5}{|c|}{ MBP, Mean Arterial Pressure; HR, Heart Rate; RR, Respiratory Rate; Sp02, Saturation of Pulse Oxygen; } \\
\hline \multicolumn{5}{|c|}{$\begin{array}{l}\mathrm{PH} \text {, Potential of Hydrogen; } \mathrm{PCO}_{2} \text {, Partial Pressure of Carbon Dioxide; } \mathrm{P} / \mathrm{F}, \mathrm{PaO} 2 / \mathrm{FiO} 2 ; \mathrm{Hb} \text {, } \\
\text { Hemoglobin. }\end{array}$} \\
\hline
\end{tabular}

\section{Outcome Of Extubation}

There were 13 episodes of extubation failure (34.2\%), 12 of them need reintubation and one death during ventilation due to the serious sepsis. Median time to reintubation was $18 \mathrm{~h}$. NIV was used infrequently after extubation $(6 / 38 ; 15.8 \%)$ and only 1 case use before reintubation $(1 / 6 ; 16.7 \%)$. Mean duration of mechanical ventilation was 31.3h (range 26.9-131.1h). Weaning failure was associated with longer ventilation duration (153 vs. $29 \mathrm{~h}, p<0.05$ )and hospital stay (26 vs. 13 days, $p<0.05$ ).

\section{Risk factors of extubation failure in MG with thymoma after thymectomy}

Analysis the risk factors for extubation failure was in Table 4. Based on previous studies and our result, risk factors that may associated with extubation failure (including age, surgical procedure, phrenic never or diaphragm injury, PTMC, HR, RR, SpO2) were analyzed by univariate analysis. After processing, the factors remained was surgical procedure, $\mathrm{RR}, \mathrm{HR}$ and $\mathrm{SpO}_{2}$. 
Table 4

Risk factors of extubation failure by univariate model

\begin{tabular}{|llllll|}
\hline Variables & $\boldsymbol{\beta}$ & Wald & OR & $95 \% \mathrm{Cl}$ & P-value \\
\hline Age & -0.053 & 3.482 & 0.948 & $0.896,1.003$ & 0.062 \\
\hline Surgical procedure (OT) & 1.812 & 5.410 & 6.125 & $1.330,28.207$ & 0.020 \\
\hline Phrenic never or diaphragm injury & 1.414 & 3.822 & 4.114 & $0.996,16.987$ & 0.051 \\
\hline PTMC & 2.288 & 6.138 & 9.857 & $1.613,60.243$ & 0.013 \\
\hline Atelectasis and/or pneumonia. & 2.357 & 8.510 & 10.556 & $2.167,51.420$ & 0.004 \\
\hline HR & 0.063 & 5.968 & 1.065 & $1.013,1.120$ & 0.015 \\
\hline RR & 0.250 & 5.168 & 1.284 & $1.035,1.594$ & 0.023 \\
\hline SpO 2 & -0.356 & 6.298 & 0.694 & $0.522,0.923$ & 0.012 \\
\hline $\begin{array}{l}\text { OT, open transsternal; PTMC, Postoperative Myasthenic Crisis; HR, Heart Rate; RR, Respiratory Rate; } \\
\text { SpO2, Saturation of Pulse Oxygen. }\end{array}$ & & & & & \\
\hline
\end{tabular}

\section{Discussion}

Our study found that extubation failure is common in T-MG patients $(13 / 38,34.2 \%)$, especially suffered from PTMC (6/8,75\%), which was, which was similar to the previous studies $23,24,26$. Transsternal thymectomy, phrenic never or diaphragm injury and postoperative pulmonary complications may increase the risk of extubation. Besides, preextubation vital signs $\left(\mathrm{HR}, \mathrm{RR}, \mathrm{SpO}_{2}\right)$ instead of blood gases did correlate with extubation outcome. Patients who experienced failure of extubation had much longer ICU and hospital stays.

It has been proved the thymus has a key role in inducing AChR antibodies production, however the specific mechanism remains unclear[19]. Guidelines[20] suggest extended thymectomy is recommended for T-MG patients, which means that maximal thymic tissue and the surrounding anterior mediastinal fat tissue have to be removed[21], and minimize the surgical procedure like VAST are preferred[22]. In our study, all T-MG patients received extended thymectomy. VAST was performed in 78.9\%(30/38) patients, 3 of them were converted to OT due to invasion of the great vessels. OT was performed in 8 patients and was associated with much more blood loose ( $800 \mathrm{vs.} 100 \mathrm{ml}, p<0.001)$, longer operation time (300 vs. $170 \mathrm{~min}, p<0.001)$ and a higher risk of extubation failure (63.6\% vs. $22.2 \%, p=0.039)$ and longer duration of ICU stay (16 vs.22 days, $p=0.013)$, which is similar with previous studies[3, 23, 24]. Although VATS can reduce trauma and had significantly less blood loss, less pain, faster recovery[25-27], possible perioperative complications related to myasthenia must be involved in surgical planning, especially respiratory impairment. Respiratory insufficiency due to diaphragmatic and intercostal muscle weakness is a major challenge. 
It was similar in our study, 28 (73.7\%) patients have locally advanced tumors (Masaoka stage II-IV) and the mean size of thymomas is considerable as $5 \mathrm{~cm}$ (range, $3-8 \mathrm{~cm}$ ).

As a result, the incident of phrenic never or diaphragm injured is $39.5 \%$ in all patients, which can the diaphragmatic function deteriorated sharply worsen the and is associated with greater risk of extubation failure $(61.5 \%$ vs. $28 \%, p=0.045)$.

It has been recommended that special attention have to paid to respiratory function in MG patients. Previous studies[28, 29] suggested that extubation can be attempted when passed SBT and have a MIP $>-20 \mathrm{cmH}_{2} \mathrm{O}$. However, our study shows that MIP was lower in the extubation group, the difference did not reach statistical significance (38 vs. $33, p=0.061$ ). Similar to the previous studies[30], few patients may have poor FVC results because of poor cooperation and technical reasons, even after their respiratory muscles have recovered adequate strength. For MG patients, the use of accessory muscles or paradoxical movement of the abdomen may be the first sign of respiratory muscle fatigue, which will cause an increase in respiratory rate with shallower tidal volume breaths. In our study, $76.9 \%(10 / 13)$ of extubation failure patients were in the late stage (Osserman Classification IIb -IV) with bulbar symptoms. Even though all patients had passed SBT, the ones who have higher RR, $\mathrm{HR}$, and lower $\mathrm{SpO}_{2}$ after $\mathrm{SBT}$ associate with have higher ratio extubation $(\mathrm{P}<0.05)$. But there was no significant difference in $\mathrm{PCO}_{2}$ or $P / F$ before weaning $(P>0.05)$. Nonetheless, we observed that muscle strength would not have significant clinical improvement immediately after surgery, the variability of MIP and RR over time probably reflecting the changes for MG patients.

Additionally, PTMC is frequently associated with respiratory insufficiency and can be induced by many factors including a history of crisis, infection, chronic respiratory dysfunction, even surgery[30, 31]. In the present cohort, 9 patients (21.1\%) experienced PTMC and was an independent adverse prognostic factor for extubation failure $(p=0.020)$. Respiratory complications also can affect the prognosis of PTMC and prolonged ventilation or reintubation. In our study, 9 patients with atelectasis, 2 with pneumonia, and 5 patients suffered both, which were associated with an increased likelihood of extubation failure $(\mathrm{p}=$ 0.020). Overall, atelectasis was present in all extubate failure patients $(13 / 13)$. This finding is concordant with Rubinstein's multicenter study[15], all extubate failure patients have atelectasis $(n=7)$ and indicate atelectasis as a risk factor of reintubation among 26 episodes of PTMC. Thus, strategies aimed at preventing pneumonia are needed to improve PTMC therapy and early extubation. Interventions that reportedly prevent pneumonia especially during ventilation include suction, chest physiotherapy, early mobilization, and an optimal PEEP must be used to avoid atelectasis[28]. Timely tracheostomy may facilitate suctioning, prevent pulmonary complications and make patients feel more comfortable[32].

NIV was used in 5 patients after extubation, none of them need reintubation. On the contract, Seneviratne [33] found that the use of NIV was associated with increased chances of reintubation, and thought that may because of the untimely use. Rabinstein[34] indicated that timely NIV use after extubation can be effective in avoid reintubation in selected MC patients. Therefore, careful monitoring of the change of RR, $\mathrm{HR}, \mathrm{SpO} 2$, and using NIV before any sign of respiratory fatigue appears can be effect. 


\section{Limition}

This study is the first comprehensive analysis of predictors of extubation in T-MG patients after thymectomy. But there must be interpreted in context of limitations. First, as the nature of the retrospective observational and single center study is vulnerable to selection bias, and the results are subject to limitations. Second, the analysis is limited by the sample size and inability to stratify patients into more specific categories for comparison. Third, various physicians treated and evaluated the patients based on personal clinical judgments. These aspects need to be evaluated in more methodologically rigorous studies in the future.

\section{Conclusions}

In this single center retrospective study, the risk of extubation failure is higher in T-MG patients who received open thymectomy, had phrenic never or diaphragm injury during the surgery and the one who develop pulmonary complications during the ventilation. Even though T-MG patients had passed SBT, a higher HRIRR and lower $\mathrm{SpO}_{2}$ after SBT also indicated high risk of failure extubation. Once failure, it is associated with considerably prolonged ICU and hospital stay. However, early institution of NIV may help achieve timely, successful extubation for T-MG patients and may have a positive impact on functional outcome.

\section{Abbreviations}

MG

myasthenia gravis; AChR:acetylcholine receptor; T-MG:thymoma-associated myasthenia gravis; PTMC:postoperative myasthenic crisis; MV:mechanical ventilation; ICU:intensive care unit; VATS:videoassisted thoracoscopic; OT:open transsternal; SBT:spontaneous breath trail; NIV:noninvasive ventilation; PEEP:positive end expiratory pressure; $\mathrm{FiO}_{2}$ :the fraction of inspired oxygen; $\mathrm{PaO}_{2}$ :arterial oxygen pressure; $\mathrm{PaCO}_{2}$ :arterial carbon dioxide pressure; RR:respiratory rate; HR:heart rate; APACHEIl:acute physiology and chronic health evaluation II; MIP:maximal inspiratory pressure.

\section{Declarations}

\section{Ethical Approval and Consent to participate}

Not applicable.

\section{Consent for publication}

Not applicable.

\section{Availability of supporting data}

The authors declare that all data supporting the findings of this study are available within the article. 


\section{Competing interests}

The authors declare that they have no competing interests.

\section{Funding}

No funding was received.

\section{Authors' contributions}

AQD made contributions to the conception and design of the study, data collecting, statistical analysis, interpretation of data and have drafted the manuscript. XL participated in study design and coordination. YZA participated in study design and coordination and revised the manuscript. All authors listed meet the authorship criteria according to the latest guidelines of the International Committee of Medical Journal Editors. All authors agree with the manuscript.

\section{Acknowledgements}

Not applicable.

\section{References}

1. Sanders DB, Guptill JT. Myasthenia gravis and Lambert-Eaton myasthenic syndrome. Continuum Lifelong Learning in Neurology. 2014;20:1413.

2. Silvestri NJ, Wolfe GI. Myasthenia gravis. In: Seminars in Neurology: 2012; 2012.

3. Watanabe A, Watanabe T, Obama T. Prognostic factors for myasthenic crisis after transsternal thymectomy in patients with myasthenia gravis. J Thorac Cardiovasc Surg. 2004;127(3):868-76.

4. Monden Y, Nakahara K, Nanjo S, Fujii Y, Matsumura A, Masaoka A, Kawashima Y. Invasive thymoma with myasthenia gravis. Cancer. 1984;54(11):2513-8.

5. Papatestas AE, Genkins G, Kornfeld P, Eisenkraft JB, Fagerstrom RP, Pozner J, Aufses AH Jr. Effects of thymectomy in myasthenia gravis. Ann Surg. 1987;206(1):79-88.

6. Grob D, Arsura EL, Brunner NG, Namba T. The course of myasthenia gravis and therapies affecting outcome. Ann N Y Acad Sci. 1987;505:472-99.

7. Gilhus NE. Myasthenia Gravis. N Engl J Med. 2016;375(26):2570-81.

8. Thomas CE, Mayer SA, Gungor Y, Swarup R, Webster EA, Chang I, Brannagan TH, Fink ME, Rowland LP. Myasthenic crisis: clinical features, mortality, complications, and risk factors for prolonged intubation. Neurology. 1997;48(5):1253-60.

9. Liu N, Liu Q, Wu X, Liu K, Vadis Q. Predictors of outcome of myasthenic crisis. Neurol Sci. 2015;36(5):801-2.

10. Valenzuela VJ, Pinochet UR, Escobar CM, Marquez AJ, Riquelme VR, Cruces RP. Ventilator-induced diaphragmatic dysfunction. Rev Chil Pediatr. 2014;85(4):491-8. 
11. Levine S, Nguyen T, Taylor N, Friscia ME, Budak MT, Rothenberg P, Zhu J, Sachdeva R, Sonnad S, Kaiser LR, et al. Rapid disuse atrophy of diaphragm fibers in mechanically ventilated humans. N Engl J Med. 2008;358(13):1327-35.

12. Frutos-Vivar F, Esteban A. Critical illness polyneuropathy: A new (or old?) reason for weaning failure. Crit Care Med. 2005;33(2):452-3.

13. Boles JM, Bion J, Connors A, Herridge M, Marsh B, Melot C, Pearl R, Silverman H, Stanchina M, Vieillard-Baron A. Weaning from mechanical ventilation. Eur Respir J. 2007;29(5):1033-56.

14. Bernardes Neto SCG, Torres R, Lima I, Resqueti VR, Fregonezi GAF. Weaning from mechanical ventilation in people with neuromuscular disease: protocol for a systematic review. BMJ Open. 2019;9(11):e029890.

15. Rabinstein AA, Mueller-Kronast N. Risk of extubation failure in patients with myasthenic crisis. Neurocrit Care. 2005;3(3):213-5.

16. Seneviratne J, Mandrekar J, Wijdicks EFM, Rabinstein AA. Predictors of extubation failure in myasthenic crisis. Arch Neurol. 2008;65(7):929.

17. Sieb JP. Myasthenia gravis: an update for the clinician. Clin Exp Immunol. 2014;175(3):408-18.

18. Liang GH, Gu ZT, Li Y, Fu JH, Fang WT. Comparison of the Masaoka-Koga and The IASLC/ITMIG Proposal for The TNM Staging Systems Based on the Chinese Alliance for Research in Thymomas (ChART)Retrospective Database. Chinese journal of lung cancer. 2016;19(7):425-36.

19. Marx A, Pfister F, Schalke B, Saruhan-Direskeneli G, Melms A, Strobel P. The different roles of the thymus in the pathogenesis of the various myasthenia gravis subtypes. Autoimmun Rev. 2013;12(9):875-84.

20. Wolfe GI, Kaminski HJ, Cutter GR. Randomized Trial of Thymectomy in Myasthenia Gravis. N Engl J Med. 2016;375(20):2006-7.

21. Comacchio GM, Marulli G, Mammana M, Natale G, Schiavon M, Rea F. Surgical Decision Making: Thymoma and Myasthenia Gravis. Thorac Surg Clin. 2019;29(2):203-13.

22. Cata JP, Lasala JD, Williams W, Mena GE. Myasthenia Gravis and Thymoma Surgery: A Clinical Update for the Cardiothoracic Anesthesiologist. J Cardiothorac Vasc Anesth. 2019;33(9):2537-45.

23. Leuzzi G, Meacci E, Cusumano G, Cesario A, Chiappetta M, Dall'armi V, Evoli A, Costa R, Lococo F, Primieri $\mathrm{P}$, et al. Thymectomy in myasthenia gravis: proposal for a predictive score of postoperative myasthenic crisis. Eur J Cardiothorac Surg. 2014;45(4):e76-88. discussion e88.

24. a AWM, a TWM, a TOM, a TMM, a HOM, a YIM, a NTM, a KKM, a TAM: Prognostic factors for myasthenic crisis after transsternal thymectomy in patients with myasthenia gravis. Journal of Thoracic \& Cardiovascular Surgery 2004, 127(3):868-876.

25. Yang Y, Dong J, Huang Y. Thoracoscopic thymectomy versus open thymectomy for the treatment of thymoma: A meta-analysis. Eur J Surg Oncol. 2016;42(11):1720-8.

26. Odaka M, Shibasaki T, Kato D, Mori S, Asano H, Yamashita M, Morikawa T. Comparison of oncological results for early- and advanced-stage thymomas: thoracoscopic thymectomy versus 
open thymectomy. Surg Endosc. 2017;31(2):734-42.

27. Zahid I, Sharif S, Routledge T, Scarci M. Video-assisted thoracoscopic surgery or transsternal thymectomy in the treatment of myasthenia gravis? Interact Cardiovasc Thorac Surg. 2011;12(1):40-6.

28. Varelas PN, Chua HC, Natterman J, Barmadia L, Zimmerman P, Yahia A, Ulatowski J, Bhardwaj A, Williams MA, Hanley DF. Ventilatory care in myasthenia gravis crisis: assessing the baseline adverse event rate. Crit Care Med. 2002;30(12):2663-8.

29. Aggarwal AN, Gupta D, Behera D, Prabhakar S, Jindal SK. Intensive respiratory care in patients with myasthenic crisis. Neurol India. 2002;50(3):348-51.

30. Juel VC. Myasthenia gravis: management of myasthenic crisis and perioperative care. Semin Neurol. 2004;24(1):75-81.

31. Ando T, Omasa M, Kondo T, Yamada T, Sato M, Menju T, Aoyama A, Sato T, Chen F, Sonobe M, et al. Predictive factors of myasthenic crisis after extended thymectomy for patients with myasthenia gravis. Eur J Cardiothorac Surg. 2015;48(5):705-9. discussion 709.

32. Liu Z, Yao S, Zhou Q, Deng Z, Zou J, Feng H, Zhu H, Cheng C. Predictors of extubation outcomes following myasthenic crisis. J Int Med Res. 2016;44(6):1524-33.

33. Seneviratne J, Mandrekar J, Wijdicks EFM, Rabinstein AA. Noninvasive Ventilation in Myasthenic Crisis. Arch Neurol. 2008;65(1):54-8.

34. Rabinstein AA, Wijdicks EFM. Weaning from the ventilator using BiPAP in myasthenia gravis. Muscle Nerve. 2003;27(2):252-3. 